\title{
Dieta de jaguatirica (Leopardus Pardalis) no sul da Amazônia brasileira
}

A fragmentação florestal e perda de habitat são os principais fatores de extinção de espécies nas florestas tropicais como a Amazônia, e os mamíferos carnívoros são os primeiros a desaparecerem por exclusão competitiva. A jaguatirica é um mesopredador de hábito alimentar oportunista com dieta generalista, o que, frente a uma paisagem cada vez mais antropizada e fragmentada, Ihe confere vantagens adaptativas em primeiro momento. Visando uma melhor compreensão da dieta de jaguatirica no sul da Amazônia, foi analisado o conteúdo estomacal de três jaguatiricas da região do município de Alta Floresta. Na composição da dieta de jaguatirica osanfíbios foram mais abundantes (50\%), seguido por mamíferos $(31.25 \%)$, répteis $(12.50 \%)$ e aves $(6.25 \%)$. Foi relatado ainda predação de um jacare (Crocodylia: Alligatoridae), e de uma preguiça (Choloepus sp.). Os resultados sugerem que a dieta de jaguatirica no sul da Amazônia está associadaà disponibilidade de recursos classificando-a como espécie oportunista generalista.

Palavras-chave: Amazônia; Mesopredadores; Dieta.

\section{Diet of Leopardus Pardalis (ocelot) in southern brazilian Amazon}

Forest fragmentation and habitat loss are the main factors in the extinction of tropical rainforests such as the Amazon, and carnivorous mammals are the first to disappear due to competitive exclusion. The ocelot is an omnipresent of opportunistic food habits with a generalist diet, which, given an increasingly anthropic and fragmented landscape, gives it adaptive advantages at first. Aiming for a better understanding of the ocelot diet in southern Amazonia, the stomach contents of three ocelots from the region of Alta Floresta were analyzed. In the composition of the ocelot diet osanfibians were more abundant (50\%), followed by mammals (31.25\%), reptiles (12.50\%) and birds (6.25\%). It was also reported predation of an alligator (Crocodylia: Alligatoridae), and of a laziness (Choloepus sp.). The results suggest that the ocelot diet in southern Amazonia is associated with the availability of resources classifying it as a generalist opportunistic species.

Keywords: Amazon; Mesopredator; Diet.

Topic: Notas Científicas

Reviewed anonymously in the process of blind peer.
Received: 02/02/2018

Approved: 20/05/2018
Cristiano de Oliveira Shuingues

Universidade do Estado de Mato Grosso, Brasil

http://lattes.cnpq.br/5639661123676902

cristiano-af12@hotmail.com

\section{Gerlane de Medeiros Costa}

Universidade do Estado de Mato Grosso, Brasil

http://lattes.cnpq.br/1535926805564494

gerlanemedeiroscosta@gmail.com

\section{Derick Victor de Souza Campos}

Universidade do Estado de Mato Grosso, Brasil

http://lattes.cnpq.br/2785712474480148

camposdvictor@gmail.com

\author{
Maicon Diego Pinto Ross \\ Universidade do Estado de Mato Grosso, Brasil \\ http://lattes.cnpq.br/7032165737809727 \\ maicon_diego10@hotmail.com \\ Reginaldo Carvalho dos Santos \\ Universidade do Estado de Mato Grosso, Brasil \\ http://lattes.cnpq.br/2622228725714785
}

carvalhotnn@gmail.com

Mendelson Guerreiro de Lima

Universidade do Estado de Mato Grosso, Brasi

http://lattes.cnpq.br/6650785434966924

mendelson@unemat.br
Referencing this:

SHUINGUES, C. O.; COSTA, G. M.; CAMPOS, D. V. S.; ROSSI, M. D. P.; SANTOS, R. C.; LIMA, M. G.. Dieta de jaguatirica (Leopardus Pardalis) no sul da Amazônia brasileira. Revista Ibero-Americana de Ciências Ambientais, v.9, n.1, p.273-278, 2018. DOI:

http://doi.org/10.6008/CBPC2179-6858.2018.001.0020 


\section{INTRODUÇÃO}

A floresta amazônica representa $40 \%$ de toda a floresta tropical remanescente no mundo (KIRBY et al., 2006), e dentre os países que a compõe, o Brasil é o maior deles, com cerca de $58 \%$ do total de sua área representada pela Amazônia (KAIMOWITZ et al., 2004). A crescente demanda mundial por commodities, além da pecuária extensiva são os principais fatores no aumento do desmatamento da floresta amazônica (FEARNSIDE et al., 2015). O desmatamento, fragmentação florestal e perda de habitat, influenciam diretamente na disponibilidade de alimentos e habitats, aumentando a competição entre espécies (CARRETE et al., 2010; TABARELLI et al., 2012), podendo causar extinção por exclusão competitiva de determinadas espécies (HERNÁNDEZ-BRITO et al., 2014). Mamíferos de grande porte, principalmente os de topo de cadeia, são os primeiros a sofrerem as consequências da perda de habitat e, consequentemente, desaparecem (CHIARELLO, 1999).Inicialmente há, para os mesopredadores, um relaxamento na competição com grandes felinos(WANG et al., 2015), o que acarreta a ocupação de nichos vagos na cadeia trófica (MCKINNEY et al., 1999; TABARELLI et al., 2012). A jaguatirica, Leopardus pardalis, é um mesopredador neotropical com ampla distribuição geográfica pela América do Sul (REIS et al., 2011). São caçadores terrícolas de hábito alimentar oportunista com dieta generalista, consumindo uma ampla variedade de presas como pequenos roedores, aves, anfíbios (REIS et al., 2011), lagartos (BIANCHI et al., 2014; MORENO et al., 2006), ocasionalmente presas de maior porte como cutias (Dasyprocta) e pacas (Cuniculus paca)(ALIAGA-ROSSEL et al., 2006; SANTOS et al., 2014), com registros de consumo de mamíferos de extrato arbóreo (MIRANDA et al., 2005; SANTOS et al., 2014).Pouco se sabe sobre a interação predador-presa na floresta amazônica, dessa forma, descrever a dieta de mesopredadores em ambientes antropizados torna-se essencial para traçar estratégias eficazes e eficientes de conservação da espécie para manutenção da biodiversidade.

\section{RELATO}

Visando uma melhor compreensão da dieta de L. pardalis no sul da Amazônia, foi analisado o conteúdo estomacal de cinco jaguatiricas adultas, todas em óbito por atropelamento em rodovias dentro dos limites do município de Alta Floresta, Mato Grosso, Boletins de Ocorrências 2014.317059 (fêmea) e 2015.77025 (macho); doações do Instituto Brasileiro do Meio Ambiente e dos Recursos Renováveis - IBAMA, conforme registros 44/09 (macho) e 01/15 (fêmea), e uma última fêmea oriunda do Canteiro de obras da UHE-Colíder (MA-0034), município de Nova Canaã-MT. Todos os espécimes deste estudo foram incorporados à Coleção Zoológica do Laboratório de Zoologia e Morfologia Animal, da Universidade do Estado de Mato Grosso, Campus de Alta Floresta. Em laboratório, foram retirados os estômagos dos cinco espécimes para quantificação e pesagem do conteúdo estomacal, a fim de obter dados de frequências e biomassa, com posterior identificação dos itens alimentares até o nível taxonômico possível. Dois espécimes estavam com estômago vazio, e dessa forma, não foram considerados na análise. A biomassa total, obtida dos três estômagos, foi de 727g, distribuídos em seis táxons distintos de cinco ordens de vertebrados (Figura 1). 

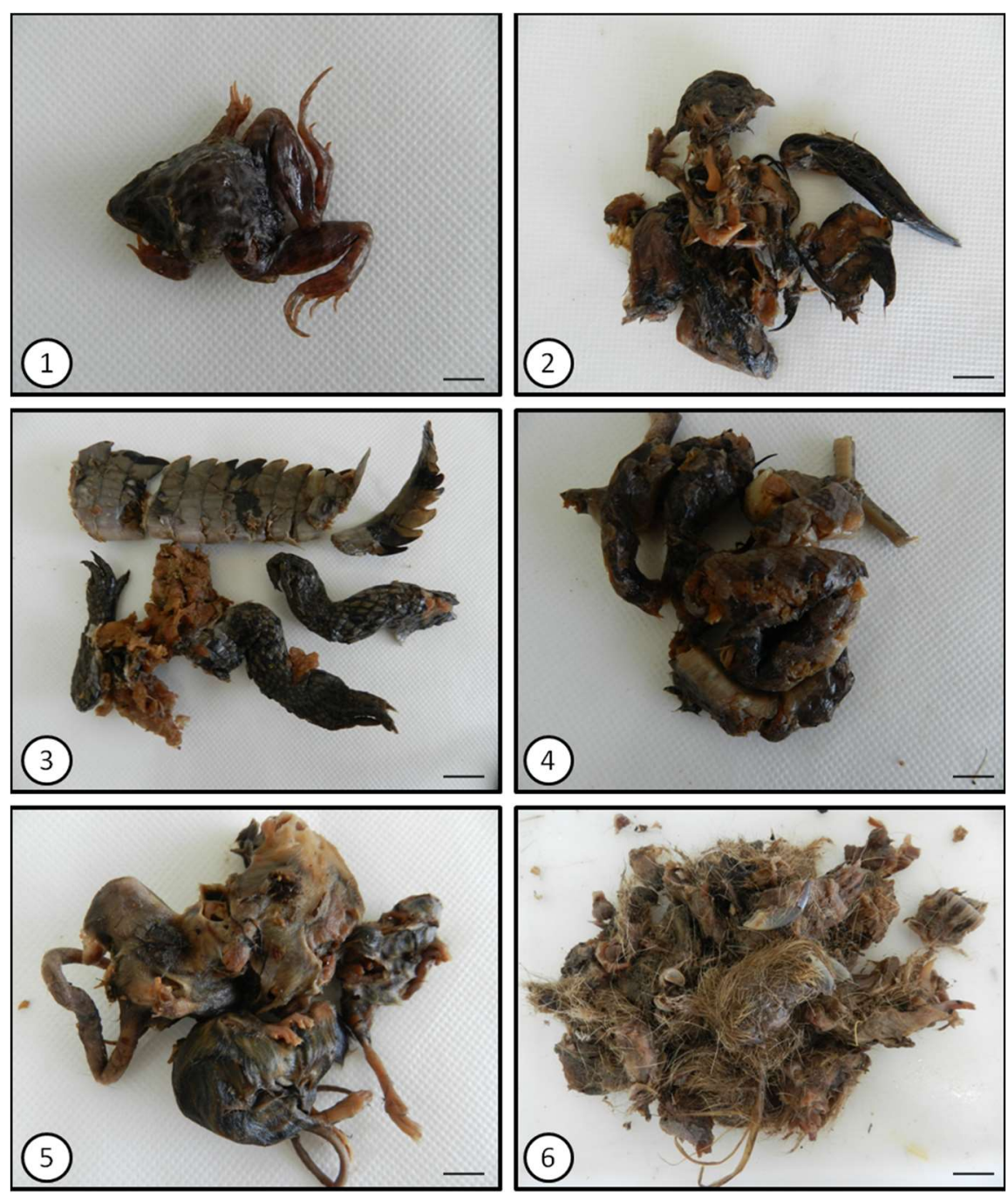

Figura 1 - Itens alimentares de L. pardalis no sul da Amazônia. 1 - Leptodactylus sp. (Anura: Leptodactylidae); 2 - Ave; 3 - Jacaré (Crocodylia: Alligatoridae); 4 - Leptodeiraannulata (Serpente: Colubridae); 5 - Roedores; 6 - preguiça (Pilosa: Megalonychidae). Barra $1 \mathrm{~cm}$.

Dentre os itens que compõe a dieta de L. pardalis no sul da Amazônia, os anfíbios foram os mais abundantes (50\%), seguido por mamíferos (31.25\%), répteis (12.50\%) e aves (6.25\%).Apesar de responderem pela maior abundânciaentre os itens alimentares, os anfíbios foram representados apenas por um único gênero Leptodactylus sp. Os mamíferos com maior representatividade foram os roedores (25\%). Quanto à biomassa, os répteis foram os de maior importância, sendo que a maior representatividade corresponde a um indivíduo da família Alligatoridae (Crocodylia). O segundo grupo na ordem de representatividade foi o dos mamíferos, dos quais uma pequena porcentagem corresponde a indivíduos da ordem Rodentia, e a maior parte corresponde a um indivíduo do gênero Choloepus (Pilosa: Megalonychidae). Aves e anfíbios representaram $13.07 \%$ e $5.23 \%$ da biomassa presente nos estômagos de jaguatirica respectivamente (Tabela 1).

Tabela 1: Itens alimentares encontrados em três estômagos de Leoparduspardalisno sul da Amazônia.

\begin{tabular}{|c|c|c|c|c|c|}
\hline & Taxon & Abund. & Abund. Rel. (\%) & Biom. (g) & Biom. Rel. (\%) \\
\hline \multirow{5}{*}{ Mammalia } & Rodentia & & & & \\
\hline & não identificado & 4 & 25 & 110 & 15.13 \\
\hline & Pilosa & & & & \\
\hline & Choloepus sp. & 1 & 6.25 & 149 & 20.49 \\
\hline & Total & 5 & 31.25 & 259 & 35.62 \\
\hline Aves & Não identificado & 1 & 6.25 & 38 & 5.23 \\
\hline
\end{tabular}




\begin{tabular}{|c|c|c|c|c|c|}
\hline \multirow{5}{*}{ Reptilia } & Crocodylia & & & & \\
\hline & Alligatoridae & 1 & 6.25 & 290 & 39.89 \\
\hline & Serpente & & & & \\
\hline & Leptodeiraannulata & 1 & 6.25 & 45 & 6.19 \\
\hline & Total & 2 & 12.5 & 335 & 46.08 \\
\hline \multirow{2}{*}{ Amphibia } & Anura & & & & \\
\hline & Leptodactylus sp. & 8 & 50 & 95 & 13.07 \\
\hline TOTAL & & 16 & 100 & 727 & 100 \\
\hline
\end{tabular}

\section{DISCUSSÃO}

A composição de anfíbios na dieta de L. pardalis, tem sido reportada como baixa e com pouca significância (BIANCHI et al., 2013), e as altas significâncias estão sempre relacionadas a pequenos mamíferos, especialmente roedores (MORENO et al. 2006, BIANCHI et al.,2007; ABREU et al., 2007). Neste estudo, os resultados diferiram do encontrado na literatura, porém, considerando a diferença de composição na dieta dos três indivíduos, os resultados confirmam o comportamento alimentar generalista e oportunista, intimamente ligado a disponibilidade de presas no ambiente. Nas análises, um único indivíduo havia consumido apenas dois táxons, ambos ligados a presença de corpos d'agua, os quais foram possíveis de identificar por que estavam em processo inicial de digestão, demonstrando recente predação.

Mamíferos de pequeno porte, especialmente da ordem Rodentia, são comumente encontrados em estômagos de L. pardalis (EMMONS, 1988), entretanto, são encontrados relatos recorrentes na literatura sobre predação de primatas (BIANCHI et al., 2007, MIRANDA et al., 2005; SANTOS et al., 2014), marsupiais (ABREL et al., 2008, MORENO et al., 2006), tamanduás (KONECNY 1989), porco-espinho e jupará (CHINCHILLA 1997), sugerindo que a espécie possua alguma habilidade para captura de presas no extrato arbóreo (ABREU et al., 2007), podendo justificar a presença da preguiça (Choloepus sp.) na composição da dieta dos espécimes avaliados. Delibes et al (2011), aponta que a predação de preguiças por jaguatirica é uma prática um tanto incomum, com poucos registros na literatura.

O consumo de repteis, principalmente lagartos, já foi reportado em outros estudos por Villa-Meza et al (2002), predação de Ctenosaura pectinata, e Bianchi et al (2010)reportando predação de Tupinambis merianae, sendo estes, reportados como principal item na dieta de jaguatirica. A predação de jacaréporL. pardalis ainda não havia sido registrada em outros estudos, sendo comumente reportado na dieta de Pantheraonca (AZEVEDO et al., 2012). A ingestão de presas cuja presença está sempre associada a presença de corpos d'agua, nesse caso jacarés e anfíbios, sugerem que L. pardalis possa utilizar matas de galeria como área de caça, conforme descrito por (EMMONS, 1987; REIS et al., 2011).

A competição é um fator que exerce pressão seletiva entre as espécies (PIANKA, 1974), neste estudo, a ingestão de presas não habituais, e.g., jacarés e preguiças, pode estar relacionada a um relaxamento na competição com grandes felinos (COVE et al., 2012), e ao mesmo tempo, aumento na competição entre mesopredadores, fazendo com que L. pardalisutilizasse um nicho trófico vago, consumindo presas não habituais.Moreno et al. (2006)sugerem que, mesmo em situações de relaxamento de competição, o tamanho das presas de jaguatirica está sempre relacionado ao seu tamanho corporal, fato observado em nossas análises onde foi registrado fragmentos de um espécime jovem de jacaré.Ainda, a predação de espécies de ambientes aquáticos pode estar refletindo a exploração de ambientes ripáriospor se tratar de áreas de 
fragmentos de florestas ainda conservadas devido à legislação que protege as áreas ao longo dos corpos de água denominadas APPs (Áreas de Preservação Permanentes). Na região sul da Amazônia extensas áreas de florestas foram convertidas em pastagense (MICHALSKI et al., 2005) ou monoculturas de soja (SILVA et al., 2018), reduzindo drasticamente a área de uso da espécie.

\section{CONSIDERAÇÕES}

Este trabalho demonstrou como a dieta de L. pardalis pode estar intimamente associada à disponibilidade de recursos no ambiente, o que explicaria a diferença na composição da dieta entre os três espécimes analisados. Os resultados reafirmam ainda o comportamento de predação oportunista e generalista de L. pardalis, o que pode Ihe conferir, em primeira impressão, maior capacidade adaptativa frente a um ambiente cada vez mais antropizado. Contudo, os resultados deste estudo, associados a estudos sobre nicho trófico das comunidades de mamíferos mesopredadores na Amazônia devem ser incentivados, uma vez que a diferenciação/segregação de nicho entre duas espécies distintas que competem ou não, pode ser fator determinante tanto na composição da dieta, quanto na permanência e perpetuação da outra em um determinado habitat (COVE et al., 2012; WANG et al.,, 2015).

\section{REFERÊNCIAS}

ALIAGA-ROSSEL, E. et al. Ocelot (Leopardus pardalis) Predation on Agouti (Dasyprocta punctata). Biotropica, v. 38, n. 5, p. 691-694, 2006.

AZEVEDO, F. C. C.; VERDADE, L. M. Predator-prey interactions: Jaguar predation on caiman in a floodplain forest. Journal of Zoology, v. 286, n. 3, p. 200-207, 2012.

BIANCHI, R. DE C. et al. Intraspecific, interspecific, and seasonal differences in the diet of three mid-sized carnivores in a large neotropical wetland. Acta Theriologica, v. 59, n. 1, p. 13-23, 2014.

BIANCHI, R. DE C.; MENDES, S. L.; JUNIOR, P. DE M. Food habits of the ocelot, Leopardus pardalis, in two areas in southeast Brazil. Studies on Neotropical Fauna and Environment, v. 45, n. 3, p. 111-119, 2010.

CARRETE, M. et al. Winners and losers in human-made habitats: Interspecific competition outcomes in two Neotropical vultures. Animal Conservation, v. 13, n. 4, p 390-398, 2010.

CHIARELLO, A. G. Effects of fragmentation of the Atlantic forest on mammal communities in south-eastern Brazil. Current, v. 89, n. 1, p. 71-82, 1999.

COVE, M. V et al. Use of camera traps to examine the mesopredator release hypothesis in a fragmented Midwestern landscape. American Midland Naturalist, v. 168, p. 456-465, 2012.

EMMONS, L. H. Comparative feeding ecology of felids in a neotropical rainforest. Behavioral Ecology and Sociobiology, v. 20, n. 4, p. 271-283, 1987.
EMMONS, L. H. A field study of ecelots (Felis pardalis) in Peru. Rev. Ecol. (Terre Vie), v. 43, 1988

FEARNSIDE, P. M.; FIGUEIREDO, A. M. R. China's influence on deforestation in brazilian Amazonia: A growing force in the state of Mato GrossoGlobal Economic Governance Initiative, 2015

HERNÁNDEZ-BRITO, D. et al. Crowding in the city: Losing and winning competitors of an invasive bird. PLoS ONE, v. 9, n. 6, 2014.

KAIMOWITZ, D. et al. Hamburger connection fuels Amazon desctruction: Cattle ranching and deforestation in Brazil's Amazon. [s.l: s.n.]

KIRBY, K. R. et al. The future of deforestation in the Brazilian Amazon. Futures, v. 38, n. 4, p. 432-453, 2006.

MCKINNEY, M. L.; LOCKWOOD, J. L. Biotic homogenization: A few winners replacing many losers in the next mass extinction. Trends in Ecology and Evolution, v. 14, n. 11, p. 450-453, 1999.

MICHALSKI, F.; PERES, C. A. Anthropogenic determinants of primate and carnivore local extinctions in a fragmented forest landscape of southern Amazonia. Biological Conservation, v. 124, n. 3, p. 383-396, 2005.

MIRANDA, J. M. D. et al. Predation on Alouatta guariba clamitans Cabrera (Primates, Atelidae) by Leopardus pardalis (Linnaeus) (Carnivora, Felidae). Revista Brasileira de Zoologia, v. 22, n. 3, p. 793-795, 2005.

MORENO, R. S.; KAYS, R. W.; SAMUDIO, R. Competitive Release in Diets of Ocelot (Leopardus Pardalis) and Puma (Puma Concolor) After Jaguar (Panthera Onca) Decline. 
Journal of Mammalogy, v. 87, n. 4, p. 808-816, 2006.

PIANKA, E. R. Niche overlap and diffuse competition.

Proceedings of the National Academy of Sciences, v. 71, $\mathrm{n}$. 5, p. 2141-5, 1974

REIS, N. R. DOS et al. Mamíferos do Brasil. 2nd. ed. Londrina, PR: [s.n.].

SANTOS, J. et al. High consumption of primates by pumas and ocelots in a remnant of the Brazilian Atlantic Forest. Brazilian Journal of Biology, v. 74, n. 3, p. 632-641, 2014.

TABARELLI, M.; PERES, C. A.; MELO, F. P. L. The "few winners and many losers" paradigm revisited: Emerging prospects for tropical forest biodiversity. Biological Conservation, v. 155, p. $136-140,2012$.

VILLA-MEZA, A.; MARTÍNEZ-MEYER, E.; LOPEZ-GONZALEZ, C. Ocelot (Leopardus pardalis) Food Habits in a Tropical Deciduous Forest of Jalisco, Mexico. The American Midland Naturalist Journal, v. 148, n. 1, p. 146-154, 2002

WANG, Y.; ALLEN, M. L.; WILMERS, C. C. Mesopredator spatial and temporal responses to large predators and human development in the Santa Cruz Mountains of California. Biological Conservation, v. 190, p. 23-33, 2015.

A CBPC - Companhia Brasileira de Produção Científica (CNPJ: 11.221.422/0001-03) detém os direitos materiais desta publicação. Os direitos referem-se à publicação do trabalho em qualquer parte do mundo, incluindo os direitos às renovações, expansões e disseminações da contribuição, bem como outros direitos subsidiários. Todos os trabalhos publicados eletronicamente poderão posteriormente ser publicados em coletâneas impressas sob coordenação da Sustenere Publishing, da Companhia Brasileira de Produção Científica e seus parceiros autorizados. Os (as) autores (as) preservam os direitos autorais, mas não têm permissão para a publicação da contribuição em outro meio, impresso ou digital, em português ou em tradução. 